\title{
Holographic entanglement entropy in boundary conformal field theory
}

\author{
En-Jui Chang, ${ }^{\dagger}$ Chia-Jui Chou, ${ }^{*}$ and Yi Yang ${ }^{*}$ \\ Department of Electrophysics, National Chiao Tung University, Hsinchu 300, Republic of China
}

(Received 7 September 2018; published 21 November 2018)

\begin{abstract}
We study the holographic entanglement entropy in a $(d+1)$-dimensional boundary quantum field theory at both the zero and finite temperature. The phase diagrams for the holographic entanglement entropy at various temperatures are obtained by solving the entangled surfaces in the different homology. We also verify the Araki-Lieb inequality and illustrate the entanglement plateau.
\end{abstract}

DOI: 10.1103/PhysRevD.98.106016

\section{INTRODUCTION}

Entanglement is a pure quantum mechanics phenomenon inherent in quantum states. It can be measured quantitatively by the entanglement entropy associated with a specified entangled region $\mathcal{A}$ located on a Cauchy slice of the spacetime by integrating out the degrees of freedom in its complementary region $\mathcal{A}^{c}$. It has been shown that the leading divergence term of the entanglement entropy is proportional to the area of the entangling boundary, i.e., the boundary of the entangled region $\mathcal{A}$. Furthermore, the finite part of the entanglement entropy contains nontrivial information about the quantum states. It is well known that the entanglement entropy of a given region and its complement are the same, $S_{\mathcal{A}}=S_{\mathcal{A}^{c}}$, for a system with only pure states. However, this is not true anymore for a system with finite temperature. The difference $\delta S_{\mathcal{A}}=S_{\mathcal{A}}-S_{\mathcal{A}^{c}}$ has been conjectured to satisfy the Araki-Lieb inequality $\left|\delta S_{\mathcal{A}}\right| \leq$ $S_{\mathcal{A} \cup \mathcal{A}^{c}}[1]$.

Calculating entanglement entropy is usually a not easy task in QFT. Remarkably, by the AdS/CFT correspondence [2-4], the holographic entanglement entropy (HEE) was proposed to be the area of the minimal entangled surface in [5-8] and was justified later on [9-12]. This prescription gives a very simple geometric picture to compute the HEE and has been widely studied for the various holographic setups, for a review see [13]. It was shown that the ArakiLieb inequality is due to the homology constraint for the entangled surface [14-16]. For certain choices of the

\footnotetext{
*Corresponding author. yiyang@mail.nctu.edu.tw phyenjui@gmail.com

agoodmanjerry.ep02g@nctu.edu.tw

Published by the American Physical Society under the terms of the Creative Commons Attribution 4.0 International license. Further distribution of this work must maintain attribution to the author(s) and the published article's title, journal citation, and DOI. Funded by SCOAP ${ }^{3}$.
}

entangled regions, there are disconnected minimal surfaces satisfying the homology constraint that leads to the famous phenomenon of the entanglement plateau [17].

On the other hand, BQFT is a quantum field theory defined on a manifold with a boundary where some suitable boundary conditions are imposed. It has important applications in the physical systems with boundaries. For example, string theory with various branes and some condensed matter systems including Hall effect, chiral magnetic effect, topology insulator, etc. Several years ago, holographic BQFT was proposed by extending the manifold where BQFT is defined to a one-dimensional higher asymptotically AdS space, i.e., the bulk manifold, with a geometric boundary $[18,19]$. The key point of holographic BQFT is thus to determine the shape of the geometric boundary in the bulk. For the simple shapes with high symmetry such as the case of a disk or half plane, many elegant results for BQFT have been obtained in [18-20]. Some interesting developments of BQFT can be found in [19-27].

Since both the entanglement entropy and BQFT can be studied holographically, it is natural to investigate the HEE in holographic BQFT. The HEE in pure AdS bulk spacetime has been studied in [28-30]. It was found that the proper boundary condition gives the orthogonal condition that requires the minimal entangled surface must be normal to the geometric boundaries if they intersect. The authors in $[28,29]$ also found an interesting phenomenon that the entanglement entropy depends on the distance to the boundary and carries a phase transition. In addition, the HEE in the $(2+1)$-dimensional bulk manifold, such as $\mathrm{AdS}_{3}$ and BTZ black hole, has been considered in [31,32].

In this work, we study the HEE in a $(d+1)$ dimensional BQFT at both the zero and finite temperatures. In the case of the zero temperature, we consider the $(d+2)$-dimensional pure AdS spacetime as the bulk manifold. We find three phases for the HEE depending on the size and the location of the entangled region $\mathcal{A}$. Our result is consistent with the conclusion in $[28,29]$. 
In the case of the finite temperature, we consider the $(d+2)$-dimensional Schwazschild-AdS black hole as the bulk manifold. The similar three phases are found for the HEE. However, due to the presence of the black hole horizon, the HEE for the entangled region $\mathcal{A}$ and its complementary region $\mathcal{A}^{c}$ are different. For BQFT at the finite temperature, entanglement entropy is mixed with the thermal entropy given by the Bekenstein-Hawking entropy $S_{\mathrm{BH}}$. We show that the Araki-Lieb inequality $\left|\delta S_{\mathcal{A}}\right| \leq S_{\mathrm{BH}}$ always holds by a directly calculation. In addition, we obtain the entanglement plateau for various sizes and locations of the entangled region $\mathcal{A}$. Because there is a new phase due to the geometric boundary, the entanglement plateau enjoys much richer structure in QFT with boundaries.

The paper is organized as follows. In Sec. II, we briefly review the holographic BQFT and present the solutions which we will use in this work. The HEE in BQFT is calculated in Sec. III. We discuss the phase structure of the HEE at both the zero and finite temperatures. We also verify the Araki-Lieb inequality and obtain the entanglement plateau. We summarize our results in Sec. IV.

\section{HOLOGRAPHIC BOUNDARY QUANTUM FIELD THEORY}

We consider a $(d+2)$-dimensional bulk manifold $\mathcal{N}$ which has a $(d+1)$-dimensional conformal boundary $\mathcal{M}$ as shown in the Fig. 1. The bulk manifold $\mathcal{N}$ is either a pure AdS spacetime, as in Fig. 1(a), or an asymptotic AdS black hole with an event horizon, as in Fig. 1(b). In addition, there is a $(d+1)$-dimensional hypersurface $\mathcal{Q}$ in $\mathcal{N}$ that intersects the conformal boundary $\mathcal{M}$ at a $d$-dimensional hypersurface $\mathcal{P}$. A BQFT is defined on $\mathcal{M}$ within the boundary $\mathcal{P}$. The hypersurface $\mathcal{Q}$ could be considered as the extension of the boundary $\mathcal{P}$ from $\mathcal{M}$ into the bulk $\mathcal{N}$

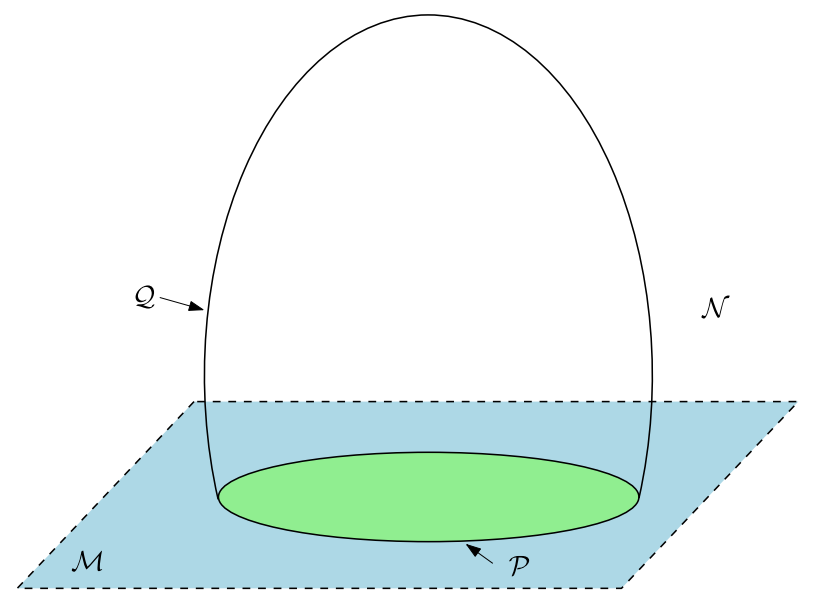

(a) Pure AdS spacetime and represents a geometric boundary of the bulk. This is our holographic setup for a BQFT living in $\mathcal{M}$ with a boundary $\mathcal{P}$.

The total action of the system is the sum of the actions of the various geometric objects and their boundary terms,

$$
S=S_{\mathcal{N}}+S_{G H}+S_{\mathcal{Q}}+S_{\mathcal{P}},
$$

where

$$
\begin{aligned}
S_{\mathcal{N}} & =\int_{\mathcal{N}} \sqrt{-g}\left(R-2 \Lambda_{\mathcal{N}}\right), \\
S_{\mathcal{Q}} & =\int_{\mathcal{Q}} \sqrt{-h}\left(R_{\mathcal{Q}}-2 \Lambda_{\mathcal{Q}}+2 K\right), \\
S_{\mathcal{M}} & =2 \int_{\mathcal{M}} \sqrt{-\gamma} K^{\prime} \\
S_{\mathcal{P}} & =2 \int_{\mathcal{P}} \sqrt{-\sigma} \theta
\end{aligned}
$$

and we have taken $16 \pi G=1$. In the total action (2.1), $S_{\mathcal{N}}$ is the action of the bulk manifold $\mathcal{N}$ with $R$ and $\Lambda_{\mathcal{N}}$ being the intrinsic Ricci curvature and the cosmological constant of $\mathcal{N} . S_{\mathcal{Q}}$ is the action of the geometric boundary $\mathcal{Q}$ with $R_{\mathcal{Q}}, \Lambda_{\mathcal{Q}}$ and $K$ being the intrinsic Ricci curvature, the cosmological constant and the extrinsic curvatures of $\mathcal{Q}$ embedded in $\mathcal{N} . S_{\mathcal{M}}$ is the action of the conformal boundary of $\mathcal{M}$ with $K^{\prime}$ being the extrinsic curvatures of $\mathcal{M}$ embedded in $\mathcal{N}$. We remark that the terms of $K$ and $K^{\prime}$ are the Gibbons-Hawking boundary terms for the boundaries $\mathcal{Q}$ and $\mathcal{M}$ of the bulk manifold $\mathcal{N}$, respectively. Finally, $S_{\mathcal{P}}$ is the common boundary term of $\mathcal{Q}$ and $\mathcal{M}$ with $\theta=\cos ^{-1}\left(n^{\mathcal{Q}} \cdot n^{\mathcal{N}}\right)$ being the supplementary angle

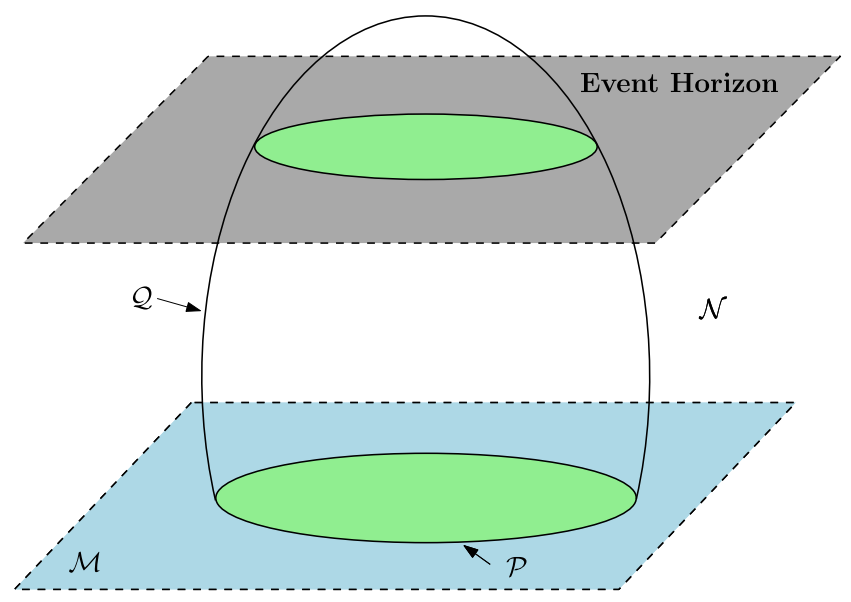

(b) AdS black hole

FIG. 1. Spacetime setup for the holographic BQFT. (a) The bulk manifold is a pure AdS spacetime. (b) The bulk manifold is an asymptotic AdS black hole with a horizon. 
between $\mathcal{Q}$ and $\mathcal{M}$, which makes a well-defined variational principle on $\mathcal{P}$. Furthermore, $g_{a b}$ denotes the metric of the bulk manifold $\mathcal{N}, h_{a b}=g_{a b}-n_{a}^{\mathcal{Q}} n_{b}^{\mathcal{Q}}$ and $\gamma_{a b}=g_{a b}-n_{a}^{\mathcal{N}} n_{b}^{\mathcal{N}}$ denote the induced metric of the boundaries $\mathcal{Q}$ and $\mathcal{M}, \sigma_{a b}$ denotes the metric of $\mathcal{P}$. Here we have defined the unit normal vectors of $\mathcal{Q}$ and $\mathcal{M}$ as $n^{\mathcal{Q}}$ and $n^{\mathcal{N}}$.

Varying $S_{\mathcal{N}}$ with $g^{a b}$ gives the equation of motion of the bulk $\mathcal{N}$,

$$
0=R_{a b}-\frac{1}{2} R g_{a b}+\Lambda_{\mathcal{N}} g_{a b}
$$

Varying $S_{\mathcal{Q}}$ with $h^{a b}$ gives the equation of motion of the geometric boundary $\mathcal{Q}$,

$$
R_{\mathcal{Q} a b}+2 K_{a b}-\left(\frac{1}{2} R_{\mathcal{Q}}+K-\Lambda_{\mathcal{Q}}\right) h_{a b}=0,
$$

which is just the Neumann boundary condition originally proposed by Takayanagi in [18] and lately generalized by Chu et al. in [29] by adding the intrinsic curvature $R_{\mathcal{Q}}$.

The crucial problem in the construction of the holographic $\mathrm{BQFT}$ is to determine the $(d+1)$-dimensional geometric boundary $\mathcal{Q}$ that satisfies the boundary condition (2.7). However, the boundary condition (2.7) is too strong to have a solution even in the pure AdS spacetime because there are more constraint equations than the degrees of freedom. In $[28,29]$, the authors proposed the following mixed boundary condition,

$$
(d-1)\left(R_{\mathcal{Q}}+2 K\right)-2(d+1) \Lambda_{\mathcal{Q}}=0 .
$$

Although it is still difficult to obtain a general solution of $\mathcal{Q}$ with the mixed boundary condition (2.8), it is possible to find solutions in some special cases. A class of solutions satisfying the mixed boundary condition (2.8) have been obtained in $[28,29]$.

In this work, instead of constructing more solutions of the geometric boundary $\mathcal{Q}$, our purpose is to study the boundary effect for the HEE in BQFT. We will thus use an almost trivial solution of $\mathcal{Q}$ that is perpendicular to the conformal boundary $\mathcal{M}$ with a simple embedding. Nevertheless, we will find rich phase structures of the HEE in our simple geometry.

We present the solutions that will be used to study the HEE in detail in the following.

\section{A. Pure AdS}

To study a $(d+1)$-dimensional BQFT at the vanishing temperature, we consider the bulk manifold $\mathcal{N}$ as the $(d+2)$-dimensional pure $\mathrm{AdS}$ spacetime $\mathrm{AdS}_{d+2}$ with the metric,

$$
d s_{\mathcal{N}}^{2}=\frac{l_{\mathrm{AdS}}^{2}}{z^{2}}\left(-d t^{2}+d z^{2}+\sum_{i=1}^{d} d x_{i}^{2}\right)
$$

where $l_{\text {AdS }}$ is the $A d S$ radius. The conformal boundary of $\operatorname{AdS}_{d+2}$ is a $(d+1)$-dimensional Minkowski spacetime located at $z=0$.

We propose a simple solution of the geometric boundary $\mathcal{Q}$ as a $(d+1)$-dimensional hepersurface embedded in the bulk manifold as

$$
d s_{\mathcal{Q}}^{2}=\frac{l_{\mathrm{AdS}}^{2}}{z^{2}}\left(-d t^{2}+d z^{2}+\sum_{i=2}^{d} d x_{i}^{2}\right),
$$

with a simple embedding $x_{1}=$ constant. The intrinsic curvature, the extrinsic curvature and the cosmological constant on $\mathcal{Q}$ can be calculated as

$$
R_{\mathcal{Q}}=-\frac{d(d+1)}{l_{\mathrm{AdS}}^{2}}, \quad K_{a b}=0, \quad \Lambda_{\mathcal{Q}}=-\frac{d(d-1)}{2 l_{\mathrm{AdS}}^{2}}
$$

It is easy to verify that the mixed boundary condition (2.8) is satisfied.

This simple solution is a special case of the solutions constructed in $[28,29]$ with $\theta=0$, i.e., the geometric boundary $\mathcal{Q}$ is perpendicular to $\mathcal{M}$ at their intersection $\mathcal{P}$.

\section{B. Schwarzschild-AdS black hole}

To study the $(d+1)$-dimensional $\mathrm{BQFT}$ at a finite temperature, we consider the bulk manifold $\mathcal{N}$ as the $(d+2)$-dimensional Schwarzschild-AdS black hole with the metric,

$$
d s_{\mathcal{N}}^{2}=\frac{l_{\mathrm{AdS}}^{2}}{z^{2}}\left(-g(z) d t^{2}+\frac{d z^{2}}{g(z)}+\sum_{i=1}^{d} d x_{i}^{2}\right),
$$

with

$$
g(z)=1-\frac{z^{d+1}}{z_{H}^{d+1}}
$$

The Hawking temperature and the Bekenstein-Hawking entropy density of the $(d+2)$-dimensional SchwarzschildAdS black hole are

$$
T=\frac{d+1}{4 \pi z_{H}}, \quad S_{\mathrm{BH}}=\frac{l_{\mathrm{AdS}}^{d} L^{d}}{4 z_{H}^{d}} .
$$

Similar to the pure AdS case, we propose a solution of the geometric boundary $\mathcal{Q}$ as a $(d+1)$-dimensional hepersurface embedded in the bulk manifold as

$$
d s_{\mathcal{Q}}^{2}=\frac{l_{\mathrm{AdS}}^{2}}{z^{2}}\left(-g(z) d t^{2}+\frac{d z^{2}}{g(z)}+\sum_{i=2}^{d} d x_{i}^{2}\right)
$$


with a simple embedding $x_{1}=$ constant. The intrinsic curvature, the extrinsic curvature and the cosmological constant on $\mathcal{Q}$ are the same as the pure AdS case,

$R_{\mathcal{Q}}=-\frac{d(d+1)}{l_{\mathrm{AdS}}^{2}}, \quad K_{a b}=0, \quad \Lambda_{\mathcal{Q}}=-\frac{d(d-1)}{2 l_{\mathrm{AdS}}^{2}}$,

which satisfy the mixed boundary condition (2.8).

\section{HOLOGRAPHIC ENTANGLEMENT ENTROPY}

Without the geometric boundary $\mathcal{Q}$, the prescription to compute the entanglement entropy holographically for the static situations has been addressed by Ryu and Takayanagi (RT) in [6,7]. It was later generalized in [8] to general states including arbitrary time dependence. In this work, we focus on the static situation.

We consider a spatial region $\mathcal{A}$ with a boundary $\partial \mathcal{A}$ lying on a Cauchy slice $\Sigma=\mathcal{A}+\mathcal{A}^{c} \subset \mathcal{M}$, with $\mathcal{A}^{c}$ the complementary part of $\mathcal{A}$, as shown in Fig. 2(a). The HEE is given by the RT formula $[6,7]$,

$S_{\mathcal{A}}=\min _{X} \frac{\operatorname{Area}\left(\mathcal{E}_{\mathcal{A}}\right)}{4 G_{N}^{(d+2)}}$

$$
X=\left\{\mathcal{E}_{\mathcal{A}}\left|\mathcal{E}_{\mathcal{A}}\right|_{\mathcal{M}}=\partial \mathcal{A} ; \exists \mathcal{R}_{\mathcal{A}} \subset \mathcal{N}, \partial \mathcal{R}_{\mathcal{A}}=\mathcal{E}_{\mathcal{A}} \cup \mathcal{A}\right\},
$$

where $\mathcal{E}_{\mathcal{A}}$ is a codimension-2 minimal surface anchored on $\partial \mathcal{A}$ in the $(d+2)$-dimensional bulk spacetime $\mathcal{N}$. The minimal surface $\mathcal{E}_{\mathcal{A}}$ is required to satisfy a homology constraint: $\mathcal{E}_{\mathcal{A}}$ is smoothly retractable to the boundary

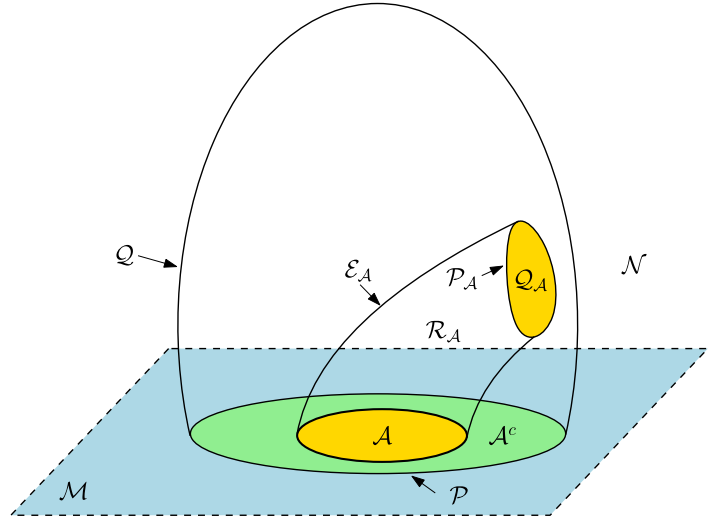

(a) Pure AdS spacetime region $\mathcal{A}$. More precisely, there exists a codimension-1 region $\mathcal{R}_{\mathcal{A}} \subset \mathcal{N}$, the so called entanglement wedge, which is bounded by the minimal surface $\mathcal{E}_{\mathcal{A}}$ and the entangled region $\mathcal{A}$ on the conformal boundary $\mathcal{M}$.

In the presence of the geometric boundary $\mathcal{Q}$, the minimal surface $\mathcal{E}_{\mathcal{A}}$ could end not only on the conformal boundary $\mathcal{M}$ but also on the geometric boundary $\mathcal{Q}$ as showed in $[28,29]$. We thus propose the following formula for the HEE in BQFT,

$$
\begin{aligned}
S_{E E}^{\mathcal{A}} & =\min _{X} \frac{\operatorname{Area}\left(\mathcal{E}_{\mathcal{A}}\right)}{4 G_{N}^{(d+2)}}, \\
X & =\left\{\mathcal{E}_{\mathcal{A}}\left|\mathcal{E}_{\mathcal{A}}\right|_{\mathcal{M}}=\partial \mathcal{A},\left.\mathcal{E}_{\mathcal{A}}\right|_{Q}=\mathcal{P}_{\mathcal{A}} ; \exists \mathcal{R}_{\mathcal{A}} \subset \mathcal{N},\right. \\
\partial \mathcal{R}_{\mathcal{A}} & \left.=\mathcal{E}_{\mathcal{A}} \cup \mathcal{A} \cup Q_{\mathcal{A}}\right\},
\end{aligned}
$$

where $\mathcal{P}_{\mathcal{A}}$ divides the geometric boundary $\mathcal{Q}$ into two parts, $\mathcal{Q}_{\mathcal{A}}$ and $\mathcal{Q}_{\mathcal{A}^{c}}$, with $\mathcal{Q}_{\mathcal{A}}$ having the same homology with $\mathcal{A}$ and $\mathcal{Q}_{\mathcal{A}^{c}}$ having the same homology with $\mathcal{A}^{c}$, as shown in Fig. 2. Requiring the boundary condition (2.8) to be smooth, $\mathcal{E}_{\mathcal{A}}$ should be orthogonal to $\mathcal{Q}$ when they intersect as showed in [28,29].

To be concrete, in this work, we consider a bulk spacetime $\mathcal{N}$ with two boundaries $\mathcal{Q}_{L, R}$ which intersect the conformal boundary $\mathcal{M}$ at $\mathcal{P}= \pm l / 2$ perpendicularly. We choose the region $\mathcal{A} \subset \mathcal{M}$ as an infinite long strip,

$x_{1} \in\left[x-\frac{a}{2}, x+\frac{a}{2}\right], \quad x_{i} \in \mathbb{R}^{d-1} \quad$ for $i=2, \cdots d$,

which preserves $(d-1)$-dimensional translation invariance in the directions $x_{i}$ for $i=2, \cdots d$.

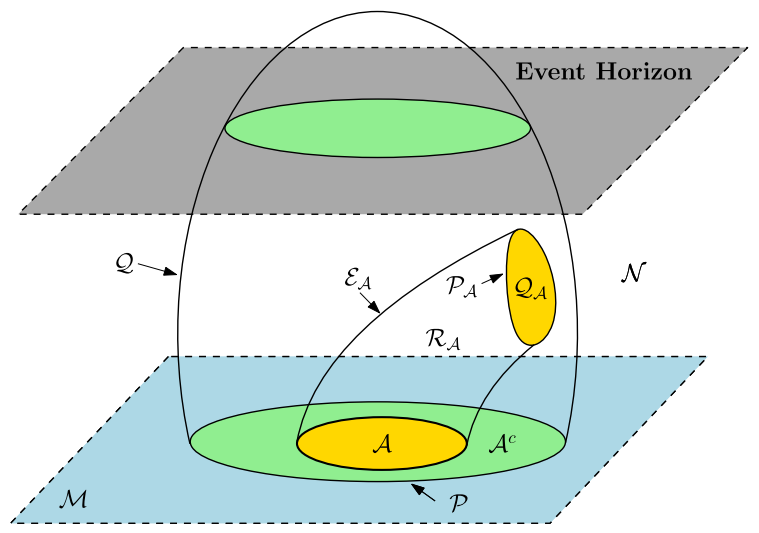

(b) AdS black hole

FIG. 2. Spacetime setup for the holographic BQFT with an entangled region $\mathcal{A}$. The minimal surface $\mathcal{E}_{\mathcal{A}}$ could end not only on the conformal boundary $\mathcal{M}$ but also on the geometric boundary $\mathcal{Q}$. (a) The bulk manifold is a pure AdS spacetime. The minimal surface for $\mathcal{A}$ and $\mathcal{A}^{c}$ have the same homology. (b) The bulk manifold is an asymptotically AdS black hole. The minimal surface for $\mathcal{A}$ and $\mathcal{A}^{c}$ have the different homologies due to the black hole horizon. 
In the static gauge, we can write down the ansatz for the minimal surface $\mathcal{E}_{\mathcal{A}}$,

$$
z=z\left(x_{1}\right), \quad z\left(x \pm \frac{a}{2}\right)=0, \quad z(x)=z_{0},
$$

$z^{\prime}(x)=0$,

where $x_{1}=x$ is the turning point of the minimal surface $\mathcal{E}_{\mathcal{A}}$.

For a general $(d+2)$-dimensional bulk metric,

$$
\begin{aligned}
d s^{2} & =-g_{t t}(z) d t^{2}+\sum_{i=1}^{d} g_{i i}(z) d x_{i}^{2}+g_{z z}(z) d z^{2}, \\
i & =1, \cdots d,
\end{aligned}
$$

the size $a$ and the HEE $S_{E E}^{\mathcal{A}}$ of the entangled region $\mathcal{A}$ can be calculated as

$$
\begin{aligned}
a= & 2 z_{0} \int_{0}^{1} d v\left[\frac{g_{11}\left(z_{0} v\right)}{g_{z z}\left(z_{0} v\right)}\left(\frac{\tilde{g}^{2}\left(z_{0} v\right)}{\tilde{g}^{2}\left(z_{0}\right)}-1\right)\right]^{-1 / 2}, \\
S_{E E}^{\mathcal{A}}= & \frac{l_{\text {AdS }}^{d} L^{d-1}}{2 G_{N}^{(d+2)}} \int_{0}^{1} d v z_{0} \tilde{g}\left(z_{0} v\right) \\
& \times\left[\frac{g_{11}\left(z_{0} v\right)}{g_{z z}\left(z_{0} v\right)}\left(1-\frac{\tilde{g}^{2}\left(z_{0}\right)}{\tilde{g}^{2}\left(z_{0} v\right)}\right)\right]^{-1 / 2},
\end{aligned}
$$

where $v=z / z_{0}$ and

$$
\tilde{g}(z)=\sqrt{\prod_{i=1}^{d} g_{i i}(z),}
$$

and $L$ is the length of the directions in which the translation invariance is preserved,

$$
\int_{\mathbb{R}^{d-1}} d^{d-1} \mathbf{x}=L^{d-1} .
$$

Using Eqs. (3.6) and (3.7), the HEE can be solved in term of the size $a$ as $S_{E E}^{\mathcal{A}}(a)$ in principle.

\section{A. Pure AdS spacetime}

We first consider the bulk spacetime $\mathcal{N}$ as a $(d+2)$ dimensional pure AdS spacetime with the metric (2.9), and choose the geometric boundary $\mathcal{Q}$ as a $(d+1)$-dimensional hepersurface with the metric (2.10). This is dual to BQFT at the zero temperature.

In the case of pure AdS spacetime, the size $a$ can be integrated to obtain

$$
a=2 z_{0} \int_{0}^{1} \frac{v^{d} d v}{\sqrt{1-v^{2 d}}}=2 z_{0} \sqrt{\pi} \frac{\Gamma\left(\frac{d+1}{2 d}\right)}{\Gamma\left(\frac{1}{2 d}\right)} .
$$

The HEE is divergent near the boundary at $v \rightarrow 0$. We thus need to regulate the HEE by putting a small cut-off $\epsilon \ll 1$. After the regulation, the HEE can be obtained as

$S_{E E}^{\mathcal{A}}=\frac{l_{\mathrm{AdS}}^{d}}{2(d-1) G_{N}^{(d+2)}}\left[\left(\frac{L}{\epsilon}\right)^{d-1}-\left(\frac{L}{z_{0}}\right)^{d} \frac{a}{2 L}\right]$,

where the divergent term is proportional to the boundary of the entangled region $\mathcal{A}$, i.e., $S_{E E}^{\mathcal{A}} \sim L^{d-1} \sim \partial \mathcal{A}$, as expected. The remaining term is finite.

In the presence of the geometric boundaries $\mathcal{Q}_{L, R}$, the minimal surface $\mathcal{E}_{\mathcal{A}}$ could anchor on $\mathcal{Q}_{L, R}$ in addition to the conformal boundary $\mathcal{M}$. In this work, we only consider the entangled region $\mathcal{A}$ being a simple connected region. Under this consideration, there are three types of the minimal surfaces which satisfy the homology constraint, as shown in Fig. 3. If the minimal surface $\mathcal{E}_{\mathcal{A}}$ is connected and only anchors on the conformal boundary $\mathcal{M}$, the entanglement wedge $\mathcal{R}_{\mathcal{A}}$ takes the shape of the sunset as shown in Fig. 3(a). If the minimal surface $\mathcal{E}_{\mathcal{A}}$ is disconnected and each part anchors on the different geometric boundaries $\mathcal{Q}_{L, R}$ in addition to the conformal boundary $\mathcal{M}$, the entanglement wedge $\mathcal{R}_{\mathcal{A}}$ takes the shape of the sky as shown in Fig. 3(b). Finally, if the minimal surface $\mathcal{E}_{\mathcal{A}}$ is disconnected and both part anchor on the same geometric boundary in addition to $\mathcal{M}$, the entanglement wedge $\mathcal{R}_{\mathcal{A}}$ takes the shape of the rainbow as shown in Fig. 3(c).

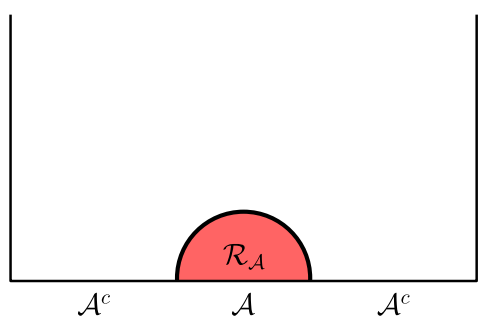

(a) Sunset

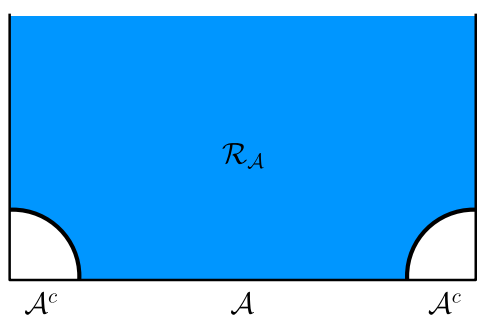

(b) Sky

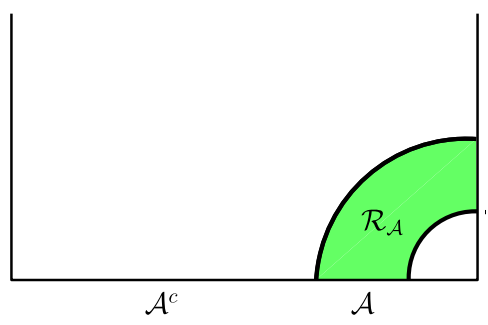

(c) Rainbow

FIG. 3. The minimal surfaces in the pure AdS bulk spacetime. (a) the entanglement wedge $\mathcal{R}_{\mathcal{A}}$ has the shape of the sunset when the entangled region $\mathcal{A}$ is very small. (b) the entanglement wedge $\mathcal{R}_{\mathcal{A}}$ has the shape of the sky when the entangled region $\mathcal{A}$ is very large. (c) the entanglement wedge $\mathcal{R}_{\mathcal{A}}$ has the shape of the rainbow when the entangled region $\mathcal{A}$ is very closed to the boundary. 
The HEE corresponding to the different minimal surfaces can be calculated as

$$
\begin{aligned}
S_{\text {sunset }}^{\mathcal{A}} & =S_{E E}^{\mathcal{A}}(a), \\
S_{\text {sky }}^{\mathcal{A}} & =\frac{1}{2} S_{E E}^{\mathcal{A}}(l-a+2|x|)+\frac{1}{2} S_{E E}^{\mathcal{A}}(l-a-2|x|),
\end{aligned}
$$

$S_{\text {rainbow }}^{\mathcal{A}}=\frac{1}{2} S_{E E}^{\mathcal{A}}(l+a-2|x|)+\frac{1}{2} S_{E E}^{\mathcal{A}}(l-a-2|x|)$.

Although each of the HEE in the above three cases is the local minimum, the global minimum depends on the size $a$ and the location $x$ of the entangled region $\mathcal{A}$.

For a small enough $\mathcal{A}$, the entanglement wedge $\mathcal{R}_{\mathcal{A}}$ takes the shape of the sunset. While for a large enough $\mathcal{A}$, the entanglement wedge $\mathcal{R}_{\mathcal{A}}$ takes the shape of the sky. If the location of the region $\mathcal{A}$ is very close to one of the geometric boundaries $\mathcal{Q}_{L, R}$, the minimal surface $\mathcal{E}_{\mathcal{A}}$ would be inclined to the boundary and break into two parts, the entanglement wedge $\mathcal{R}_{\mathcal{A}}$ will take the shape of the rainbow as shown in Fig. 3(c).

The HEE transfers among the three phases as the size $a$ and the location $x$ of the entangled region $\mathcal{A}$ varying, which correspond to the quantum phase transitions at the zero temperature in the dual BQFT. The phase diagram is shown in Fig. 4 (We set $l=1 / 2$ in the figures of this paper). At the middle, $x=0$, there is a critical value $a_{0}$ for the size of the entangled region $\mathcal{A}$. For $a<a_{0}$, the entanglement wedge $\mathcal{R}_{\mathcal{A}}$ takes the shape of the sunset, while for $a>a_{0}$, the minimal surface $\mathcal{E}_{\mathcal{A}}$ breaks into two parts and the entanglement wedge $\mathcal{R}_{\mathcal{A}}$ takes the shape of the sky. When $x$ is away from the middle, the critical value decreases until it reaches the triple critical points at $\left( \pm x_{t}, a_{t}\right)$ where a new phase, in which the entanglement wedge $\mathcal{R}_{\mathcal{A}}$ takes the shape of the rainbow, emerges due to the effect of the

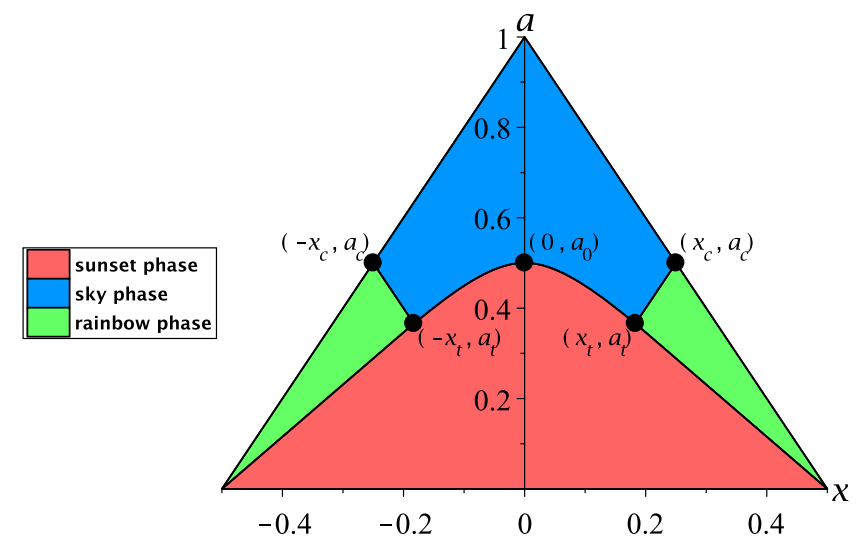

FIG. 4. Phase Diagram of the holographic entanglement entropy in the pure AdS bulk spacetime. Different phases are marked with the different colors. boundary $\mathcal{Q}$. When $|x|$ is beyond the critical points at $\left( \pm x_{c}\right.$, $a_{c}$ ), the sky phase disappears, the sunset phase and the rainbow phase compete until $x$ reaches the boundaries at $x= \pm l / 2$.

In the pure AdS case, it is easy to see that, for a entangled region $\mathcal{A}$, and its complementary $\mathcal{A}^{c}$, the associated minimal surfaces $\mathcal{E}_{\mathcal{A}}$ and $\mathcal{E}_{\mathcal{A}^{c}}$ have the same homology. Therefore, $\mathcal{A}$ and $\mathcal{A}^{c}$ share the same minimal surface $\mathcal{E}_{\mathcal{A}}=\mathcal{E}_{\mathcal{A}^{c}}$, as well as the same HEE $S_{E E}^{\mathcal{A}}=S_{E E}^{\mathcal{A}^{c}}$.

\section{B. Schwarzschild-AdS black hole}

We next consider the bulk spacetime $\mathcal{N}$ as a $(d+2)$ dimensional Schwarzschild-AdS spacetime with the metric (2.12), and choose the geometric boundary $\mathcal{Q}$ as a $(d+1)$ dimensional hypersurface embeded in $\mathcal{N}$ with the metric (2.15). This is dual to BQFT at the finite temperature. The temperature in BQFT is identified with the Hawking temperature of the black hole by the holographic correspondence. The temperature and the entropy density of the black hole were given in Eq. (2.14).

In the case of AdS black hole spacetime, the size $a$ of the entangled region $\mathcal{A}$ can be expressed as the following integral

$$
a=2 z_{0} \int_{0}^{1} \frac{v^{d} d v}{\sqrt{\left(1-(b v)^{d+1}\right)\left(1-v^{2 d}\right)}},
$$

where we have defined the parameter $b=z_{0} / z_{H}$, which measures how close the minimal surface $\mathcal{E}_{\mathcal{A}}$ is from the horizon.

Similar to the pure AdS case, the HEE is divergent near the boundary at $v \rightarrow 0$ and we need to regulate it by putting a small cut-off $\epsilon$. After the regulation, the HEE can be obtained as

$$
\begin{aligned}
S_{E E}= & \frac{l_{\mathrm{AdS}}^{d}}{2(d-1) G_{N}^{(d+2)}}\left(\frac{L}{\epsilon}\right)^{d-1}-\frac{1}{(d-1) G_{N}^{(d+2)}} \frac{S_{\mathrm{BH}}}{L}\left[\frac{a}{b^{d}}\right. \\
& -b z_{0} \int_{0}^{1} \frac{(d-3) v}{\sqrt{\left(1-(b v)^{d+1}\right)\left(1-v^{2 d}\right)}} d v \\
& \left.-b z_{0} \int_{0}^{1} \frac{(d+3) v^{2 d+1}}{\sqrt{\left(1-(b v)^{d+1}\right)\left(1-v^{2 d}\right)}} d v\right]
\end{aligned}
$$

where the first term is divergent and is proportional to the boundary of the region $\mathcal{A}$ as the same as in the pure $\operatorname{AdS}$ case. The remaining terms in the square brackets are finite.

In the small size limit $a \rightarrow 0$, the turning point $z_{0} \rightarrow 0$ as well, so that the parameter $b=z_{0} / z_{H} \rightarrow 0$. The HEE thus reduces to

$$
\begin{aligned}
S_{E E} & \simeq \frac{l_{\mathrm{AdS}}^{d}}{2(d-1) G_{N}^{(d+2)}}\left(\frac{L}{\epsilon}\right)^{d-1}-\frac{a}{(d-1) G_{N}^{(d+2)} b^{d}} \frac{S_{\mathrm{BH}}}{L} \\
& =\frac{l_{\mathrm{AdS}}^{d}}{2(d-1) G_{N}^{(d+2)}}\left[\left(\frac{L}{\epsilon}\right)^{d-1}-\left(\frac{L}{z_{0}}\right)^{d} \frac{a}{2 L}\right],
\end{aligned}
$$


which is exact the same as in the pure AdS case as it should be. While for a finite size $a$, the HEE in the black hole case dramatically deviates from that in the pure AdS case.

In the case of the black hole spacetime, the associated minimal surfaces $\mathcal{E}_{\mathcal{A}}$ and $\mathcal{E}_{\mathcal{A}^{c}}$ for the entangled region $\mathcal{A}$ and its complementary $\mathcal{A}^{c}$ have different homology due to the presence of the black hole horizon, so that the HEE $S_{E E}^{\mathcal{A}}$ for a region $\mathcal{A}$ is generically not the same as the HEE $S_{E E}^{\mathcal{A}^{c}}$ for its complementary $\mathcal{A}^{c}$. This is the crucial difference between the cases of the AdS black hole and the pure AdS spacetime.

As in the pure AdS case, there are three types of the minimal surfaces depending on the size $a$ and the location $x$ of the region $\mathcal{A}$, and similarly for its complementary $\mathcal{A}^{c}$, as shown in Fig. 5. The HEE corresponding to the different minimal surfaces $\mathcal{E}_{\mathcal{A}}$ can be calculated as

$$
\begin{aligned}
S_{\text {sunset }}^{\mathcal{A}} & =S_{E E}^{\mathcal{A}}(a), \\
S_{\text {sky }}^{\mathcal{A}} & =\frac{1}{2} S_{E E}^{\mathcal{A}}(l-a+2|x|)+\frac{1}{2} S_{E E}^{\mathcal{A}}(l-a-2|x|)+S_{\mathrm{BH}},
\end{aligned}
$$

$S_{\text {rainbow }}^{\mathcal{A}}=\frac{1}{2} S_{E E}^{\mathcal{A}}(l+a-2|x|)+\frac{1}{2} S_{E E}^{\mathcal{A}}(l-a-2|x|)$.
The HEE corresponding to the minimal surfaces $\mathcal{E}_{\mathcal{A}^{c}}$ can be calculated as

$$
\begin{aligned}
S_{R-\text { sunset }}^{\mathcal{A}^{c}} & =S_{E E}^{\mathcal{A}}(a)+S_{\mathrm{BH}}, \\
S_{R-\text { sky }}^{\mathcal{A}^{c}} & =\frac{1}{2} S_{E E}^{\mathcal{A}}(l-a+2|x|)+\frac{1}{2} S_{E E}^{\mathcal{A}}(l-a-2|x|),
\end{aligned}
$$

$S_{R-\text { rainbow }}^{\mathcal{A}^{c}}=\frac{1}{2} S_{E E}^{\mathcal{A}}(l+a-2|x|)+\frac{1}{2} S_{E E}^{\mathcal{A}}(l-a-2|x|)+S_{\mathrm{BH}}$.

For a small enough $\mathcal{A}$, hence the large enough $\mathcal{A}^{c}$, the minimal surface $\mathcal{E}_{\mathcal{A}}$ only anchors on $\partial \mathcal{N}$, and the entanglement wedge $\mathcal{R}_{\mathcal{A}}$ takes the shape of the sunset, similar to the case of the pure AdS; while the minimal surface $\mathcal{E}_{\mathcal{A}^{c}}$ includes both $\mathcal{E}_{\mathcal{A}}$ and the black hole horizon, and the entanglement wedge $\mathcal{R}_{\mathcal{A}^{c}}$ takes the shape of the reversedsunset, as shown in Figs. 5(a) and 5(d). For a large enough $\mathcal{A}$, hence the small enough $\mathcal{A}^{c}$, the minimal surface $\mathcal{E}_{\mathcal{A}}$ breaks into two parts plus the horizon and the entanglement wedge $\mathcal{R}_{\mathcal{A}}$ takes the shape of the sky; while the minimal surface $\mathcal{E}_{\mathcal{A}^{c}}$ is $\mathcal{E}_{\mathcal{A}}$ minus the horizon, and the entanglement wedge $\mathcal{R}_{\mathcal{A}^{c}}$ takes the shape of the reversed-sky, as shown in Figs. 5(b) and 5(e). When the location of the entangled region $\mathcal{A}$ is very close to one of the geometric boundaries, the minimal surface $\mathcal{E}_{\mathcal{A}}$ would be inclined to the boundary

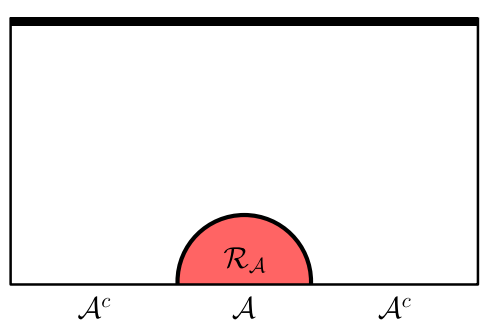

(a) Sunset

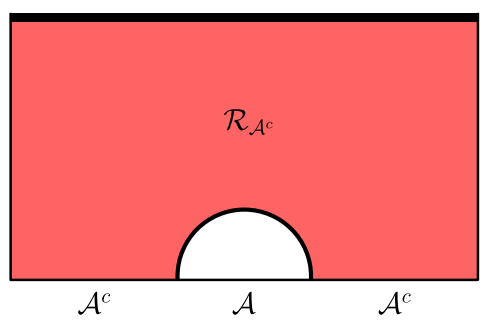

(d) Reversed-sunset

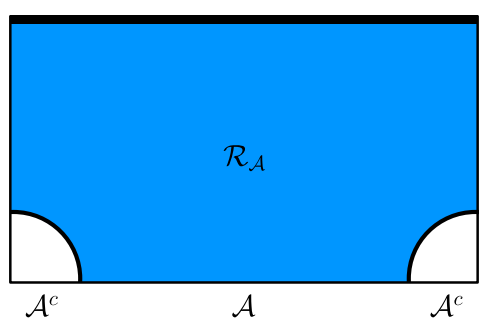

(b) Sky



(e) Reversed-sky

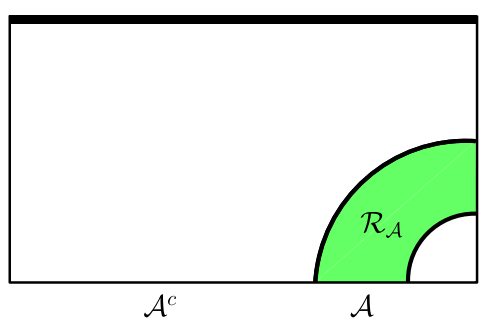

(c) Rainbow

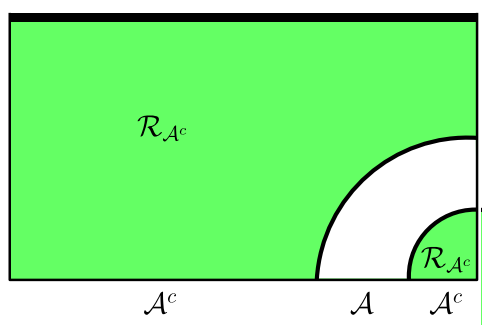

(f) Reversed-rainbow

FIG. 5. The minimal surfaces in the asymptotically AdS black hole bulk spacetime. The thick black line at the top indicates the black hole horizon. (a/d) the entanglement wedge $\mathcal{R}_{\mathcal{A}} / \mathcal{R}_{\mathcal{A}^{c}}$ has the shape of the sunset/reversed-sunset when the entangled region $\mathcal{A}$ is very small. (b/e) the entanglement wedge $\mathcal{R}_{\mathcal{A}} / \mathcal{R}_{\mathcal{A}^{c}}$ has the shape of the sky/reversed-sky when the entangled region $\mathcal{A}$ is very large. (c/f) the entanglement wedge $\mathcal{R}_{\mathcal{A}} / \mathcal{R}_{\mathcal{A}^{c}}$ has the shape of the rainbow/reversed-rainbow when the entangled region $\mathcal{A}$ is very closed to the boundary. 


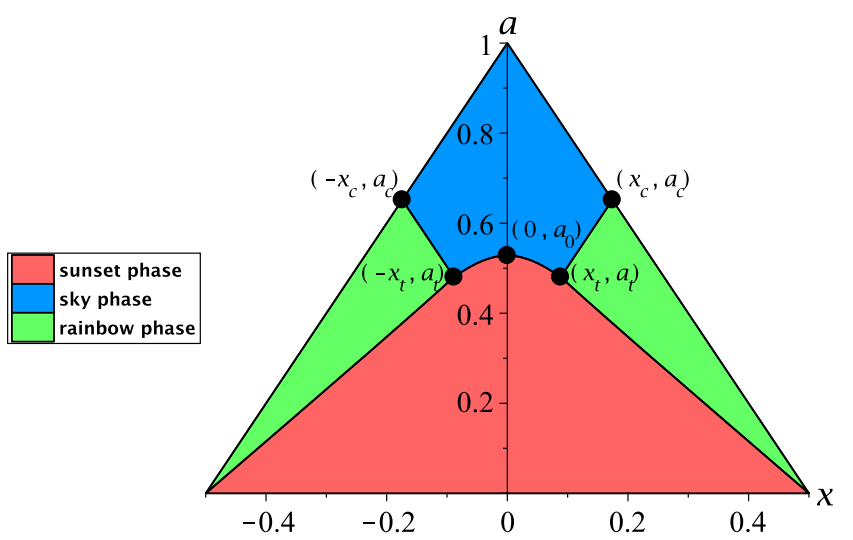

FIG. 6. Phase Diagram of the holographic entanglement entropy $S_{E E}^{\mathcal{A}}$ for a entangled region $\mathcal{A}$ in the Schwarzschild-AdS blackhole bulk spacetime with the horizon $z H=1.5$.

and the entanglement wedge $\mathcal{R}_{\mathcal{A}}$ takes the shape of the rainbow; while the minimal surface $\mathcal{E}_{\mathcal{A}^{c}}$ is $\mathcal{E}_{\mathcal{A}}$ plus the horizon and the entanglement wedge $\mathcal{R}_{\mathcal{A}^{c}}$ takes the shape of the reversed-rainbow as shown in Figs. 5(c) and 5(f). However, the positions of the phase transitions among the three phases are usually different for $\mathcal{A}$ and $\mathcal{A}^{c}$, that makes the full phase structure for $\mathcal{A}$ and $\mathcal{A}^{c}$ rather complicated.
The HEE transfers among the different phases corresponding to the phase transitions at the finite temperature in the dual BQFT. These phase transitions are the mixture of the quantum phase transition and the thermal phase transition.

Figure 6 shows the phase diagram of the HEE for the entangled region $\mathcal{A}$ at the horizon $z_{H}=1.5$, i.e., at the temperature $T=0.212$ in the dual BQFT. At the middle, $x=0$, there is a critical value $a_{0}$ for the size of the region $\mathcal{A}$. For $a<a_{0}$, the entanglement wedge $\mathcal{R}_{\mathcal{A}}$ takes the shape of the sunset, while for $a>a_{0}, \mathcal{E}_{\mathcal{A}}$ breaks into two parts plus the horizon and the entanglement wedge $\mathcal{R}_{\mathcal{A}}$ takes the shape of the sky. When $x$ is away from the center at $x=0$, the critical value decreases until it reaches the triple critical points at $\left( \pm x_{t}, a_{t}\right)$ where a new phase, in which the entanglement wedge $\mathcal{R}_{\mathcal{A}}$ takes the shape of the rainbow, emerges due to the effect of the boundary $\mathcal{Q}$. When $|x|$ is beyond the critical points at $\left( \pm x_{c}, a_{c}\right)$, the sky phase disappears, the sunset phase and the rainbow phase compete until $x$ reaches the boundaries at $x= \pm l / 2$.

The phase diagram of the HEE for the region $\mathcal{A}$ in the black hole case has the similar structure as which in the pure AdS case, but with different critical points $\left( \pm x_{t}, a_{t}\right)$ and $\left( \pm x_{c}, a_{c}\right)$. Figures 7(a)-7(d) show the critical points and the phase boundaries in the phase diagrams of the

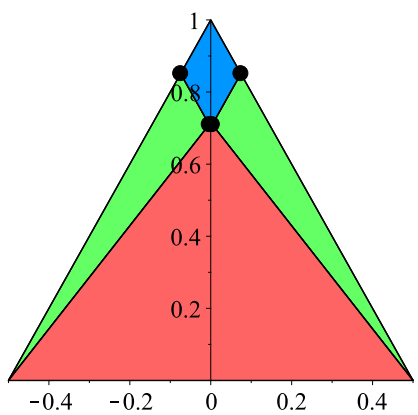

(a) $z_{H}=0.6$

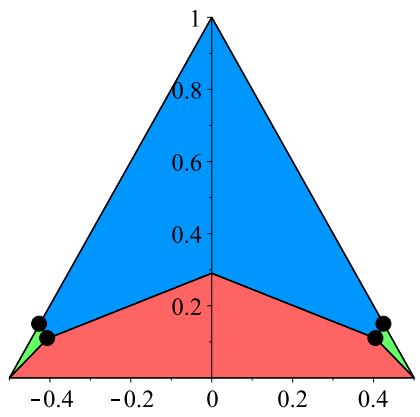

(e) $z_{H}=0.6$

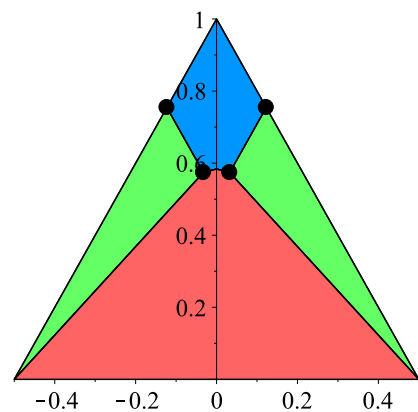

(b) $z_{H}=1$

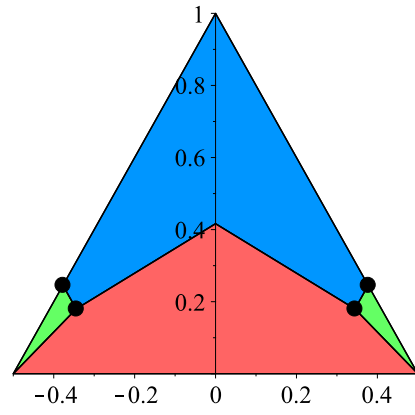

(f) $z_{H}=1$

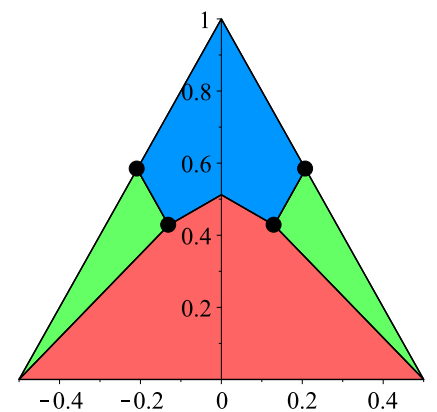

(c) $z_{H}=2$

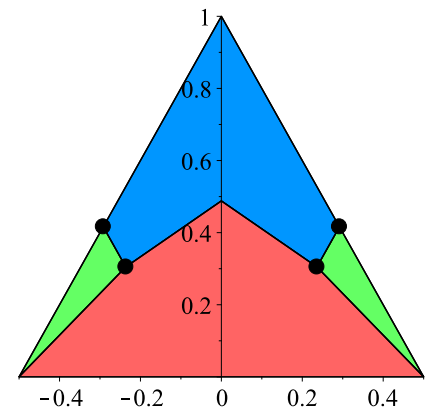

(g) $z_{H}=2$



(d) $z_{H}=9$

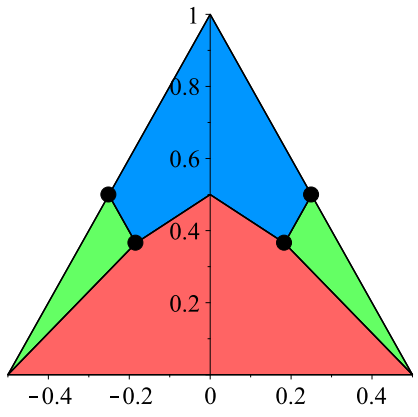

(h) $z_{H}=9$

FIG. 7. The phase diagrams of the holographic entanglement entropy in the Schwarzschild-AdS black hole with different horizons. i.e., different temperatures. The critical points $\left( \pm x_{t}, a_{t}\right)$ and $\left( \pm x_{c}, a_{c}\right)$ change with the temperatures. (a, b, c, d) The holographic entanglement entropy $S_{E E}^{\mathcal{A}}$ for the entangled region $\mathcal{A}$. (e, f, g, h) The holographic entanglement entropy $S_{E E}^{\mathcal{A}^{c}}$ for its supplementary region $\mathcal{A}^{c}$. For the large horizon or low temperature, the phase diagrams of $S_{E E}^{\mathcal{A}}$ and $S_{E E}^{\mathcal{A}^{c}}$ approach to each other and become the same as the phase diagram in the pure AdS bulk spacetime. 


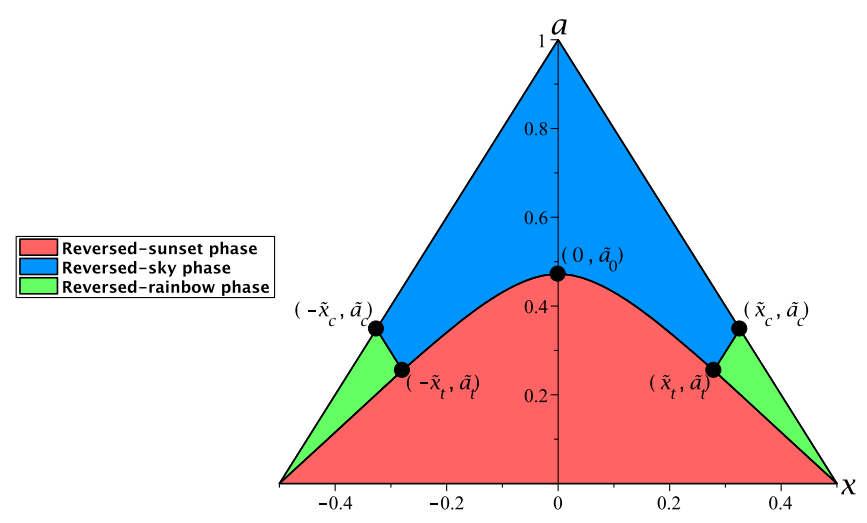

FIG. 8. Phase Diagram of the holographic entanglement entropy $S_{E E}^{\mathcal{A}^{c}}$ for a entangled region $\mathcal{A}$ in the Schwarzschild-AdS blackhole bulk spacetime with the horizon $z H=1.5$.

HEE $S_{E E}^{\mathcal{A}}$ at the different temperatures. In the lowtemperature limit, i.e., the large $z_{H}$, the phase diagram in the black hole case is asymptotic to the phase diagram in the pure AdS case as expected. While in the hightemperature limit, i.e., the small $z_{H}$, both the sky and the rainbow phases shrink to zero, only the sunset phase survives.

The behaviors of the HEE in these two limits are easy to understand from the viewpoint of the holographic principle. In the low-temperature limit, the horizon is far away from the conformal boundary $\mathcal{M}$ at $z=0$ where the BQFT lives, so that the horizon hardly affects the shape of the minimal surface. In addition, the Bekenstein-Hawking entropy density in Eq. (2.14) approaches to zero in this limit and can be neglected. Therefore, the system in the low-temperature limit is the same as that in the pure AdS spacetime.

On the other hand, in the high-temperature limit, the horizon is very close to the conformal boundary so that the rainbow phase is impossible. In addition, the BekensteinHawking entropy density is divergent in this limit so that the sky phase, which includes the horizon, is disfavored. Therefore, only the sunset phase exists in the high-temperature limit.

The HEE of $\mathcal{A}^{c}$ has the similar behavior with the critical points $\left( \pm \tilde{x}_{t}, \tilde{a}_{t}\right)$ and $\left( \pm \tilde{x}_{c}, \tilde{a}_{c}\right)$. The phase diagram for the HEE of $\mathcal{A}^{c}$ is shown in Fig. 8. At the middle $x=0$, there is a critical value $\tilde{a}_{0}$. For $a<\tilde{a}_{0}$, the entanglement wedge $\mathcal{R}_{\mathcal{A}^{c}}$ takes the shape of the reversed-sunset; while for $a>\tilde{a}_{0}, \mathcal{E}_{\mathcal{A}^{c}}$ breaks into two parts plus the horizon and the entanglement wedge $\mathcal{R}_{\mathcal{A}^{c}}$ takes the shape of the reversedsky. When $x$ is away from the middle at $x=0$, the critical value decreases until it reaches the triple critical points at $\left( \pm \tilde{x}_{t}, \tilde{a}_{t}\right)$ where a new phase, in which the entanglement wedge $\mathcal{R}_{\mathcal{A}^{c}}$ takes the shape of the reversed-rainbow, emerges due to the effect of the boundary $\mathcal{Q}$. When $|x|$ is beyond another critical points at $\left( \pm \tilde{x}_{c}, \tilde{a}_{c}\right)$, the reversedsky phase disappears, and the reversed-sunset phase and the reversed-rainbow phase compete until $x$ reaches the boundaries at $x= \pm l / 2$.

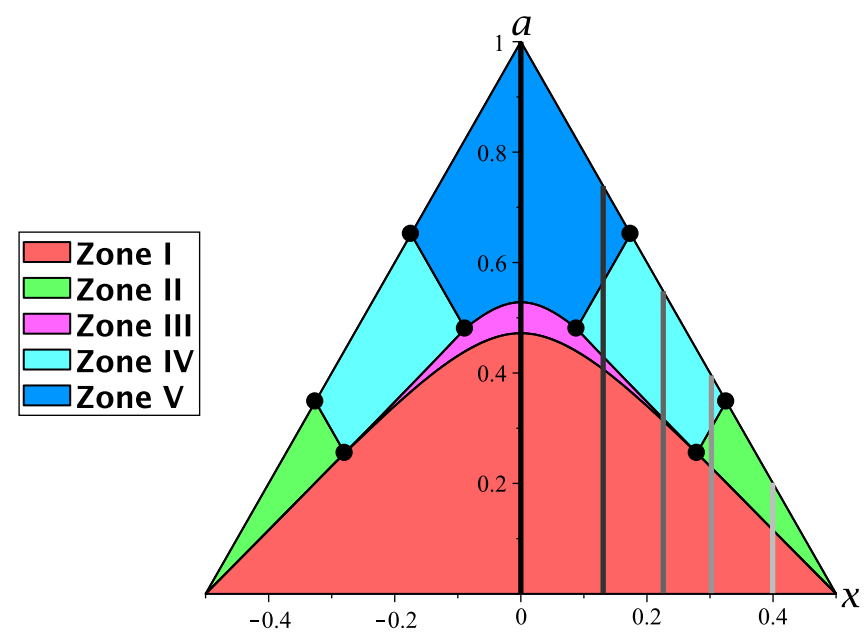

FIG. 9. The phase diagrams of the holographic entanglement entropy $S_{E E}^{\mathcal{A}}$ and $S_{E E}^{\mathcal{A}^{c}}$ for the entangled region $\mathcal{A}$ and its complementary $\mathcal{A}^{c}$ are combined together. There are five zones, marked with different colors, that are associated to the different phases. The vertical lines represent the different phase transition tracks at the different location $x=0,0.13,0.23,0.30,0.40$.

However, the critical points $\left( \pm \tilde{x}_{t}, \tilde{a}_{t}\right)$ and $\left( \pm \tilde{x}_{c}, \tilde{a}_{c}\right)$ for $\mathcal{A}^{c}$ shift with the temperature in the opposite way of $\left( \pm x_{t}, a_{t}\right)$ and $\left( \pm x_{c}, a_{c}\right)$ by a similar argument for $\mathcal{A}$. In the low-temperature limit with $z_{H} \rightarrow \infty$, the system is the same as that in the pure AdS spacetime. While in the hightemperature limit with $z_{H} \rightarrow 0$, only the reversed-sky phase exists. The critical points and the phase boundaries in the phase diagrams of the HEE $S_{E E}^{A^{c}}$ at the different temperatures are shown in Figs. 7(e)-7(h).

\section{Entanglement plateau}

It was conjectured that the HEE satisfies the Araki-Lieb inequality

$$
\left|\Delta S_{E E}^{\mathcal{A}}\right|=\left|S_{E E}^{\mathcal{A}}-S_{E E}^{\mathcal{A}^{c}}\right| \leq S_{\mathrm{BH}},
$$

in the holographic BQFT. To show that, we explore the HEE for $\mathcal{A}$ and $\mathcal{A}^{c}$ in more details by plotting their phase diagrams together in the Fig. 9. In the phase diagram, there are five zones as marked in the plot. The associated phase in each zone is listed in the Table I.

TABLE I. The shapes of $\mathcal{R}_{\mathcal{A}}$ and $\mathcal{R}_{\mathcal{A}^{c}}$ in the different zones of the phase diagram in Fig. 9. The values of $\Delta S_{E E}^{\mathcal{A}}=S_{E E}^{\mathcal{A}}-S_{E E}^{\mathcal{A}^{c}}$ for different zones are listed at the right column of the table.

\begin{tabular}{lccc}
\hline \hline Zone & $\mathcal{A}$ & $\mathcal{A}^{c}$ & $\Delta S_{E E}^{\mathcal{A}}$ \\
\hline I & Sunset & Reversed-sunset & $-S_{\mathrm{BH}}$ \\
II & Rainbow & Reversed-rainbow & $-S_{\mathrm{BH}}$ \\
III & Sunset & Reversed-sky & $\left(-S_{\mathrm{BH}}, S_{\mathrm{BH}}\right)$ \\
IV & Rainbow & Reversed-sky & $\left(-S_{\mathrm{BH}}, S_{\mathrm{BH}}\right)$ \\
V & Sky & Reversed-sky & $+S_{\mathrm{BH}}$ \\
\hline \hline
\end{tabular}




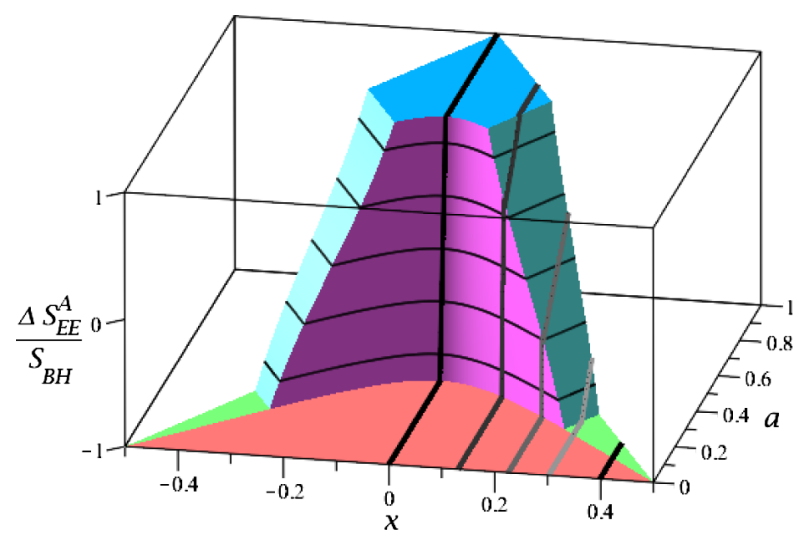

(a) 3d entanglement plateau

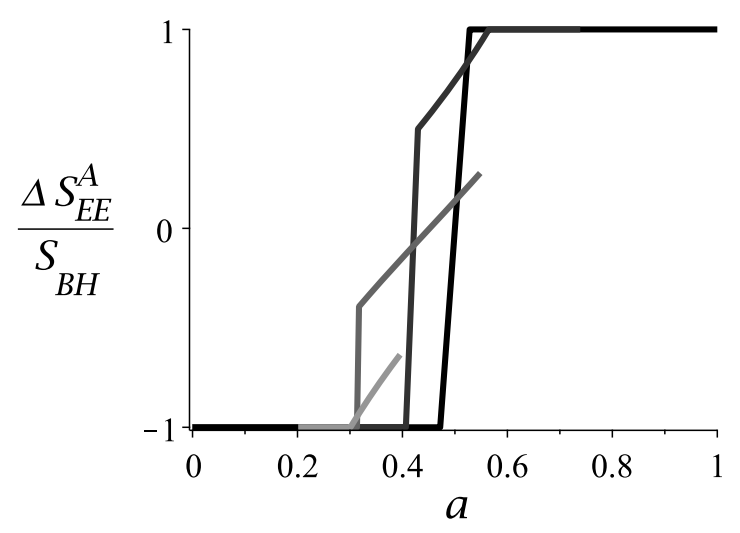

(b) Entanglement plateau at different $x$

FIG. 10. (a) The three-dimensional plot of the holographic entanglement plateau vs the location $x$ and the size $a$ of the entangled region $\mathcal{A}$. The different phase transition tracks at the different location $x=0,0.13,0.23,0.30,0.40$ are outlined and plotted in (b).

We see that $\left|\Delta S_{E E}^{\mathcal{A}}\right|=S_{\mathrm{BH}}$ for the zones I, II and V. This induces the well-known entanglement plateau. $\Delta S_{E E}^{\mathcal{A}}$ at different location $x$ is plotted in Fig. 10(b). For $|x| \leq x_{t}$, by increasing the size $a, \Delta S_{E E}^{\mathcal{A}}$ goes through the zones I-III-V and plots the typical entanglement plateau. For $x_{t} \leq|x| \leq x_{c}$, by increasing the size $a, \Delta S_{E E}^{\mathcal{A}}$ goes through the zones I-IIIIV-V and plots the plateau with a defected corner. For $x_{c} \leq|x| \leq \tilde{x}_{t}$, by increasing the size $a, \Delta S_{E E}^{\mathcal{A}}$ goes through the zones I-III-IV with the upper plateau disappearing. For $\tilde{x}_{t} \leq|x| \leq \tilde{x}_{c}$, by increasing the size $a, \Delta S_{E E}^{\mathcal{A}}$ goes through the zones I-II-IV. Finally, for $\tilde{x}_{c} \leq|x| \leq l / 2$, by increasing the size $a, \Delta S_{E E}^{\mathcal{A}}$ goes through the zones I-II and always takes the constant value $-S_{\mathrm{BH}}$. The threedimensional graph of the entanglement plateau is plotted in Fig. 10(a).

\section{SUMMARY}

In this paper, we studied the HEE in a $(d+1)$ dimensional holographic BQFT. We considered two simple solutions for the geometric boundary $\mathcal{Q}$ embedded in the $(d+2)$-dimensional bulk manifolds in the holographic BQFT. The AdS $\mathrm{A}_{d+2}$ bulk manifold corresponds to BQFT at the zero temperature, and the $(d+2)$-dimensional Schwarzschild-AdS black hole bulk manifold corresponds to BQFT at the finite temperature.

We generalized the Ryu and Takayanagi formula by including the geometric boundaries and calculated the HEE in both cases. For the pure AdS spacetime, we found three phases depending on the size and the location of the entangled region $\mathcal{A}$. We obtained the phase diagram of the HEE in the holographic BQFT. It is easy to see that the HEE $S_{E E}^{\mathcal{A}}$ for a region $\mathcal{A}$ is always the same as the HEE $S_{E E}^{\mathcal{A}^{c}}$ for its complementary $\mathcal{A}^{c}$. For the Schwarzschild-AdS black hole spacetime, we found that the HEE $S_{E E}^{\mathcal{A}}$ is generically not the same as the HEE $S_{E E}^{\mathcal{A}^{c}}$ due to the homology constraint. Three new phases were found for the region $\mathcal{A}^{c}$. We obtained the phase diagrams of the HEE for both $\mathcal{A}$ and $\mathcal{A}^{c}$ and showed that both of them are asymptotic to that in the pure $\operatorname{AdS}$ case in the lowtemperature limit as expected.

Furthermore, we verified the Araki-Lieb inequality $\left|\Delta S_{E E}^{\mathcal{A}}\right|=\left|S_{E E}^{\mathcal{A}}-S_{E E}^{\mathcal{A}^{c}}\right| \leq S_{\mathrm{BH}}$ and obtained the entanglement plateau by combining the phase diagrams of the HEE for both $\mathcal{A}$ and $\mathcal{A}^{c}$ together. We plotted the threedimensional entanglement plateau vs the size $a$ and the location $x$ of the entangled region $\mathcal{A}$.

\section{ACKNOWLEDGMENTS}

We would like to thank Chong-Sun Chu and Rong-Xin Miao for useful discussions. This work is supported by the Ministry of Science and Technology (MOST 106-2112-M009 -005 -MY3) and in part by National Center for Theoretical Science (NCTS), Taiwan. 
[1] H. Araki and E. H. Lieb, Entropy Inequalities, in Inequalities (Springer, New York, 2002), pp. 47-57.

[2] J. M. Maldacena, The large $N$ limit of superconformal field theories and supergravity, Int. J. Theor. Phys. 38, 1113 (1999); Adv. Theor. Math. Phys. 2, 231 (1998).

[3] E. Witten, Anti-de Sitter space and holography, Adv. Theor. Math. Phys. 2, 253 (1998).

[4] S. S. Gubser, I. R. Klebanov, and A. M. Polyakov, Gauge theory correlators from noncritical string theory, Phys. Lett. B 428, 105 (1998).

[5] M. Srednicki, Entropy, and Area, Phys. Rev. Lett. 71, 666 (1993).

[6] S. Ryu and T. Takayanagi, Holographic Derivation of Entanglement Entropy from AdS/CFT, Phys. Rev. Lett. 96, 181602 (2006).

[7] S. Ryu and T. Takayanagi, Aspects of holographic entanglement entropy, J. High Energy Phys. 08 (2006) 045.

[8] V. E. Hubeny, M. Rangamani, and T. Takayanagi, A covariant holographic entanglement entropy proposal, J. High Energy Phys. 07 (2007) 062.

[9] D. V. Fursaev, Proof of the holographic formula for entanglement entropy, J. High Energy Phys. 09 (2006) 018.

[10] M. Headrick, Entanglement Renyi entropies in holographic theories, Phys. Rev. D 82, 126010 (2010).

[11] H. Casini, M. Huerta, and R. C. Myers, Towards a derivation of holographic entanglement entropy, J. High Energy Phys. 05 (2011) 036.

[12] A. Lewkowycz and J. Maldacena, Generalized gravitational entropy, J. High Energy Phys. 08 (2013) 090.

[13] M. Rangamani and T. Takayanagi, Holographic entanglement entropy, Lect. Notes Phys. 931, 1 (2017).

[14] M. Headrick and T. Takayanagi, A Holographic proof of the strong subadditivity of entanglement entropy, Phys. Rev. D 76, 106013 (2007).

[15] T. Azeyanagi, T. Nishioka, and T. Takayanagi, Near extremal black hole entropy as entanglement entropy via $\operatorname{AdS}(2) / C F T(1)$, Phys. Rev. D 77, 064005 (2008).

[16] D. D. Blanco, H. Casini, L.-Y. Hung, and R. C. Myers, Relative entropy and holography, J. High Energy Phys. 08 (2013) 060.

[17] V. E. Hubeny, H. Maxfield, M. Rangamani, and E. Tonni, Holographic entanglement plateaux, J. High Energy Phys. 08 (2013) 092.
[18] T. Takayanagi, Holographic Dual of BCFT, Phys. Rev. Lett. 107, 101602 (2011).

[19] M. Nozaki, T. Takayanagi, and T. Ugajin, Central charges for BCFTs and holography, J. High Energy Phys. 06 (2012) 066.

[20] M. Fujita, T. Takayanagi, and E. Tonni, Aspects of AdS/BCFT, J. High Energy Phys. 11 (2011) 043.

[21] D. V. Fursaev, Quantum entanglement on boundaries, J. High Energy Phys. 07 (2013) 119.

[22] K. Jensen and A. O'Bannon, Holography, entanglement entropy, and conformal field theories with boundaries or defects, Phys. Rev. D 88, 106006 (2013).

[23] J. Estes, K. Jensen, A. O'Bannon, E. Tsatis, and T. Wrase, On holographic defect entropy, J. High Energy Phys. 05 (2014) 084.

[24] K. Jensen and A. O'Bannon, Constraint on Defect and Boundary Renormalization Group Flows, Phys. Rev. Lett. 116, 091601 (2016).

[25] D. V. Fursaev and S. N. Solodukhin, Anomalies, entropy and boundaries, Phys. Rev. D 93, 084021 (2016).

[26] C. Berthiere and S. N. Solodukhin, Boundary effects in entanglement entropy, Nucl. Phys. B910, 823 (2016).

[27] A. F. Astaneh and S. N. Solodukhin, Holographic calculation of boundary terms in conformal anomaly, Phys. Lett. B 769, 25 (2017).

[28] R.-X. Miao, C.-S. Chu, and W.-Z. Guo, New proposal for a holographic boundary conformal field theory, Phys. Rev. D 96, 046005 (2017).

[29] C.-S. Chu, R.-X. Miao, and W.-Z. Guo, On new proposal for holographic BCFT, J. High Energy Phys. 04 (2017) 089.

[30] A. F. Astaneh, C. Berthiere, D. Fursaev, and S. N. Solodukhin, Holographic calculation of entanglement entropy in the presence of boundaries, Phys. Rev. D 95, 106013 (2017).

[31] J. Erdmenger, M. Flory, and M.-N. Newrzella, Bending branes for DCFT in two dimensions, J. High Energy Phys. 01 (2015) 058.

[32] J. Erdmengera, M. Florya, C. Hoyosb, M.-N. Newrzellaa, and J. M. S. Wu, Entanglement entropy in a holographic Kondo model, Fortschr. Phys. 64, 109 (2016). 